\title{
Arte y lenguaje: flujos y tensiones en la educación
}

\author{
(Art and Language: Flows and Tensions in Education)
}

\section{Juan Crisóstomo IzaguirRe Ruíz, Universidad de Sonora, Sonora, México}

\section{Volumen 2, número 1}

Junio 2017

p. $189-205$

Este número se publicó el 30 de junio de 2017

ISSN: 2448-5942, doi: https://doi.org/10.36799/el.v2i1.55

Conferencia presentada en el

VII Congreso Internacional de Investigación en

Didáctica de la Lengua y la Literatura y IX Foro Nacional sobre la enseñanza de la Literatura “Josefina de Ávila Cervantes". Evento organizado por el Departamento de Letras y Lingüística de la Universidad de Sonora y El Colegio de Bachilleres del Estado de Sonora.

Hermosillo, Sonora, México., del 2 al 4 de noviembre de 2016.

\section{Cómo citar este artículo:}

Izaguirre Ruíz, J. C. (2017). Arte y lenguaje: flujos y tensiones en la educación. Estudios גambda. Teoría y práctica de la didáctica en Lengua y Literatura., 2(1), 189-205. https://doi.org/10.36799/el.v2i1.55

Derechos de autor: El autor o autores conservan en todo momento sus derechos morales y patrimoniales sobre la obra; la obra no se puede alterar, transformar o ampliar; siempre debe reconocerse la autoría del documento referido. Ninguna de las modalidades de los documentos publicados en Estudios $\lambda$ ambda. Teoría y práctica de la didáctica en lengua y literatura tienen fines comerciales de naturaleza alguna.

Los contenidos de este artículo están bajo una licencia de Creative Commons Atribución No Comercial- Sin Derivadas 4.0 Internacional @(ब) 


\title{
Arte y lenguaje: flujos y tensiones en la educación
}

\author{
(Art and Language: Flows and Tensions in Education)
}

JUAN CRISÓSTOMO IZAGUIRRE Ruíz ${ }^{1}$

\section{RESUMEN}

En este texto se presenta una reflexión crítica sobre las concepciones de arte y lenguaje que prevalecen en el currículo de Educación Básica (EB), así como en el perfil de docentes según el currículo de formación en los niveles de preescolar, primaria y secundaria. Para dar contexto se recurre a las coordenadas trazadas por la evolución de la especie y del desarrollo humano. En la convergencia de filogenia y ontogenia se sitúan arte y lenguaje como conquistas de y entre los hombres. En este marco se reconoce la doble naturaleza de dichos objetos, como productos de la evolución y como fuentes de aprendizaje en el desarrollo. Esta reflexión crítica está apoyada en el análisis sobre la interpretación que se hace de arte y lenguaje en el currículo de $\mathrm{EB}$, así como de las concepciones que los docentes reproducen mediante su práctica gracias a la formación profesional que han recibido, también mediante el currículo. Un resultado de dichas concepciones es contar con objetos de enseñanza desnaturalizados y fragmentarios, con repercusión directa en el educando. Como alternativa, se propone que el sujeto - en contacto con otros sujetos- organiza su relación con la realidad a partir de procesos como percepción, apreciación, expresión, cognición, configurando una y otra vez al mundo y a sí mismo.

PALABRAS CLAVE: arte, lenguaje, educación, desarrollo, aprendizaje

\section{ABSTRACT}

In this article is presented a critical reflection about art and language conceptions which prevail in the Basic Education (BE) curriculum, as well as in the profile of teachers according to the training curriculum in preschool, primary, and secondary levels. In order to contextualize, we recur to drawn coordinates by the evolution of the species and of human development. In the convergence of phylogeny and ontogeny, art and language are situated as conquers from and between men. In this frame the double nature of such objects is recognized as products of evolution and as sources of learning in the development. This critical reflection is supported by the analysis on the interpretation of art and language in the BE curriculum as well as in the conceptions which teachers reproduce through their practice due to the professional training they have received, and also through the curriculum. A result of such conceptions is to have denatured and fragmentary teaching objects with direct repercussions on the student. As an alternative, it is proposed that the individual - in contact with other individuals- organizes his relationship with reality from processes such as perception, appreciation, expression, cognition, configuring again and again to the world and to him.

KEY WORDS: art, language, education, development, learning

\footnotetext{
${ }^{1}$ Doctor en Educación por la Universidad Autónoma de Sinaloa; Maestro en Educación por la Universidad del Valle de México; Licenciado en Lingüística por la Universidad de Sonora; Instructor de Arte, con Especialidad en Danza, por el Instituto Nacional de Bellas Artes y Literatura. Especialista en Competencias Docentes para la Educación Media Superior, por la Universidad Pedagógica Nacional y la certificación correspondiente por la Asociación Nacional de Universidades e Instituciones de Educación Superior. Es Evaluador Certificado del Desempeño Docente en educación media superior por el Instituto Nacional para la Evaluación de la Educación.
}

Izaguirre Ruiz, doi: https://doi.org/10.36799/el.v2i1.55, Volumen 2, número 1, Año 2017, ISSN: 2448-5942 


\section{INTRODUCCIÓN}

En este texto se presenta una breve reflexión crítica sobre las concepciones de arte y lenguaje que prevalecen en el currículo de Educación Básica (EB), así como entre los docentes del mismo nivel educativo, gracias principalmente al currículo en el que se han formado. Para dar contexto a la discusión se recurre a las coordenadas trazadas por la evolución de la especie y del desarrollo humano. En la convergencia de filogenia y ontogenia se sitúan arte y lenguaje como conquistas de y entre los hombres. En este marco se reconoce la doble naturaleza de dichos objetos, como productos de la evolución y como fuentes de aprendizaje en el desarrollo. Luego se presenta un análisis sobre la interpretación que se hace de ambos en el currículo de EB así como de las concepciones que los docentes reproducen gracias a la formación profesional que han recibido, también mediante el currículo.

$\mathrm{El}$ artículo concluye recuperando la complejidad que caracteriza a arte y lenguaje como objetos en la realidad y al sujeto en medio de ella. Como alternativa, se propone que el sujeto en contacto con otros sujetos- organiza su relación con la realidad a partir de procesos como percepción, apreciación, expresión, cognición, configurando una y otra vez al mundo y a sí mismo.

\section{DEFINICIONES PRELIMINARES CLÁSICAS}

El ser humano contemporáneo es resultado de la unión de dos coordenadas: la evolución de la especie y el desarrollo del individuo. En esta convergencia, filogénica y ontogénica, los individuos han generado su propia naturaleza ensamblados en la complejidad del mundo, como reflejo de la necesidad de adaptación. En la secuencia habilis-erectus-sapiens, se explica la escala evolutiva en la que conquistas espectaculares acerca del mundo - pero también sobre el sí mismo-, así como temeridades insospechadas, han sorprendido por su grandiosidad.

Arte y lenguaje son dos de estas conquistas. Producto al mismo tiempo de la evolución y del desarrollo, ambos aparecen en las diversas realidades como dos objetos de naturaleza estrictamente humana, aunque cada uno envuelto en su propia complejidad. No obstante, en la relación que los individuos establecen con ambos objetos y las formas en que éstos afectan a aquellos, hay asimetrías. Por un lado, el lenguaje ha llegado a ser un objeto de estudio científico

Iraguirre Ruiz, doi: https://doi.org/10.36799/el.v2i1.55, Volumen 2, número 1, Año 2017, ISSN: 2448-5942 
conocido ampliamente por sus fundamentos biológicos, así como por sus funciones sociales y culturales; mientras que el arte, todavía envuelto en un ingenuo desprecio platónico (Collingood, 1985; Shiner, 2004; Izaguirre Ruíz, 2016) ha sido preferentemente colocado en el ámbito de una filosofía especulativa, a través de la estética clásica o la historia del arte, con toda clase de consecuencias entre las que resaltan las de orden epistemológico ${ }^{2}$.

Gracias a la convergencia entre evolución y desarrollo, se sabe que en el plano cerebral hay una clara diferenciación entre ambos objetos. El lenguaje es parte de las funciones superiores ubicándose en el hemisferio izquierdo; mientras que el arte está asociado al desarrollo y control de funciones afectivas, y se lo ubica en el lado derecho del cerebro. Algunas de estas asimetrías se han visto reflejadas en la valoración de que gozan arte y lenguaje. El lenguaje está fuertemente asociado a la capacidad racional y ésta al lenguaje, mientras que al arte se lo vincula con el desarrollo de la sensibilidad. A menudo hay ausencia de vínculo entre la capacidad racional y la sensibilidad. Este contraste tiene un reflejo claro - y penoso-en la educación.

\section{ARTE, LENGUAJE Y EDUCACIÓN}

Puesto que arte y lenguaje han contribuido al desarrollo de la civilización, y en la medida en que ésta ha provocado transformaciones en aquellos como productos humanos, dichos objetos están incorporados formalmente en las diferentes instituciones. La educación es una de ellas; pero la valoración que se hace de cada objeto es diferente. Uno podría preguntarse si arte y lenguaje, como productos de la evolución y fundamentos del desarrollo humano, deberían ser valorados en la misma proporción. Las respuestas serían muy pronto tan variadas como las posturas existentes respecto al tipo de ser humano y de sociedad que se proyecten. Aunque quizás una buena cantidad de tales respuestas coincidirían en reconocer un conjunto de funciones especializadas a arte y lenguaje debido a su localización hemisférica.

En los sistemas educativos contemporáneos, enmarcados concretamente en el modelo económico neoliberal, es visible una asimetría en favor del lenguaje y en detrimento del arte. El

\footnotetext{
2 Una de las más visibles consecuencias de la filosófica especulativa aplicada al arte es el conflicto irresuelto entre mimesis y poiesis como definición. Cuando esta imprecisión ontológica es llevada al aula mediante una asignatura llamada Artes, se tienen escenarios educativos en los que optar por la imitación o la creación, o bien una combinación de ambas actitudes, no puede quedar a cargo de una comunidad de docentes que en el mejor de los casos reproducen el currículo en el que se han formado (Izaguirre Ruíz, 2016).
}

Izaguirre Ruiz, doi: https://doi.org/10.36799/el.v2i1.55, Volumen 2, número 1, Año 2017, ISSN: 2448-5942 
currículo de EB en México, por ejemplo, exhibe esta diferenciación de muchas formas. Una de las más evidentes es la cantidad de horas lectivas durante el ciclo escolar dedicadas a cada campo, llamados "asignaturas" en el currículo: cinco horas semanales de Español frente a dos de Artes, en secundaria. O bien la separación curricular entre ambos objetos. Todos los diseños colocan al inicio al lenguaje, mientras que el arte casi siempre va al final (SEP, 2011). Si quisiéramos interpretar esta organización no dudaríamos en observar dos elementos distantes y sin relación.

En efecto, si observamos la posición de arte y lenguaje en el currículo de EB en México, un resultado es la percepción de dos objetos distintos y separados entre sí, y a su vez del resto de las asignaturas. Más o menos como ocurre con cualquier otro plan de estudios en cualquier nivel educativo. Todo ello a pesar de propósitos integristas expresados de diferentes maneras dentro del mismo currículo.

Las disciplinas, en torno a las cuales está organizada la enseñanza, siguen vigentes y son necesarias para organizar sistemáticamente el aprendizaje del español (y de otra lengua materna, de ser el caso), las matemáticas, las ciencias naturales, las ciencias sociales, así como la filosofía en el bachillerato, entre otras. Estas disciplinas conforman el núcleo de conocimientos básicos que se requieren para la comunicación efectiva, el pensamiento lógico y la comprensión del entorno en que vivimos.

No obstante, el cambio que se plantea está orientado a fortalecer el sentido y el significado de lo que se aprende. Se propone ensanchar y hacer más sólidos el entendimiento y la comprensión de los principios fundamentales, así como de las relaciones que los contenidos guardan entre si (SEP, 2016: 13) (Énfasis añadido).

El del currículo desarticulado no es un problema nuevo en la educación. En el caso de México el fenómeno cobra vigencia gracias a la Reforma Educativa de 2013. Como parte de esta problemática, sin ser lo más relevante, la incorporación de nuevos componentes educacionales, así como una terminología nueva, otorga al currículo una complejidad particular. Así, tendríamos que preguntarnos de qué manera y en qué proporción elementos como la comunidad, la gestión escolar y la cultura docente tienen efectos en la operativización del currículo, en el marco de la implementación de la Reforma Educativa 2013. Aunque un análisis de este tipo sería atractivo,

Iraguirre Ruir, doi: https://doi.org/10.36799/el.v2i1.55, Volumen 2, número 1, Año 2017, ISSN: 2448-5942 
en este texto será considerado sólo un subcomponente del currículo y uno más de la cultura docente: el plan de estudios y el perfil docente, respectivamente.

\section{Mapa curricular de la Educación Básica}

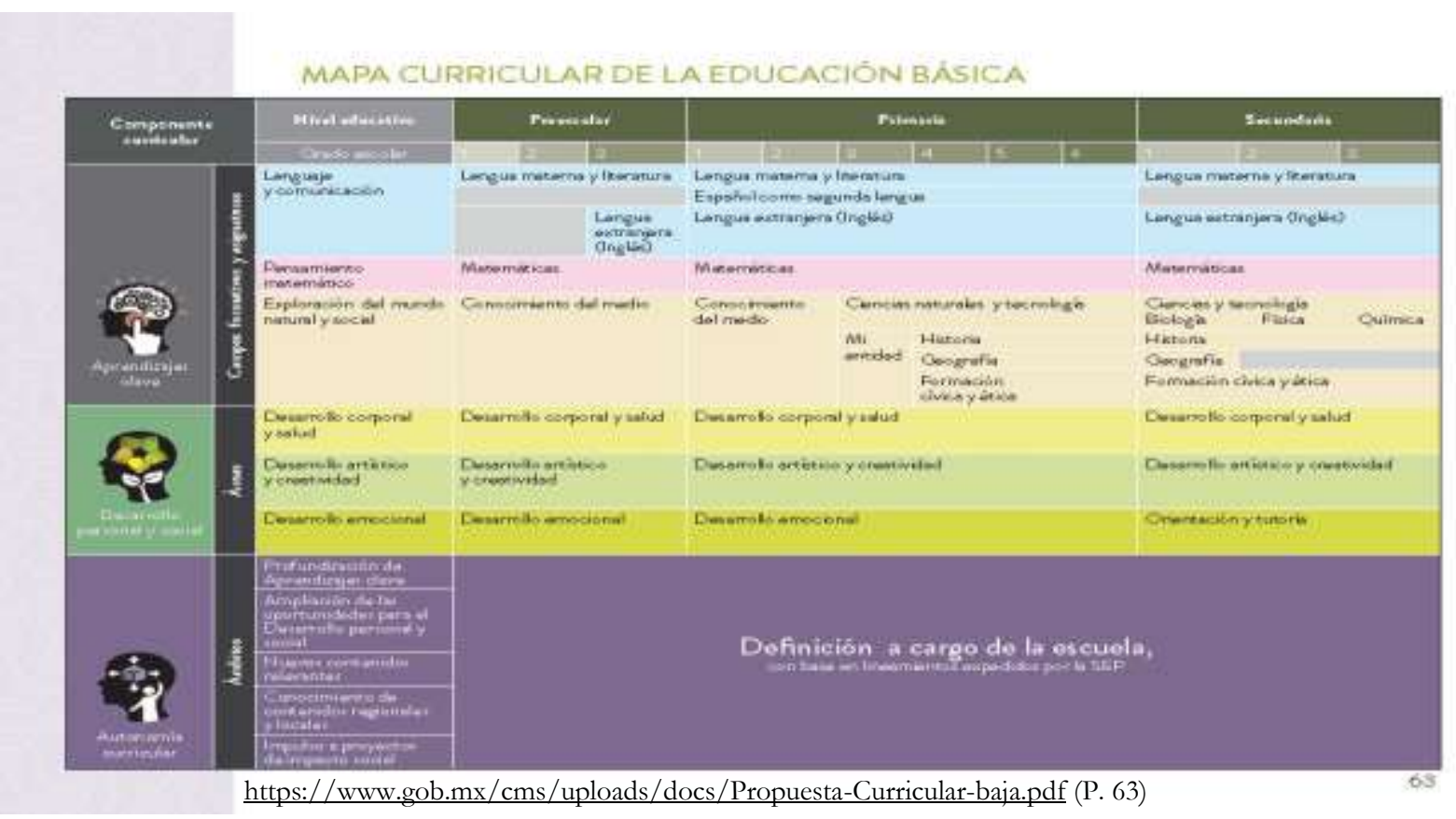

De acuerdo con la más reciente Propuesta Curricular oficial (SEP, 2016), que debe entenderse como seguimiento al Acuerdo 592 sobre la articulación de la educación básica (SEP, 2011), en el currículo se distribuyen tres grandes componentes (dispuestos en forma vertical, al extremo izquierdo del mapa curricular) atravesando los tres niveles educativos. Estos componentes son: Aprendizajes clave, Desarrollo personal y social, Autonomía curricular. Este último es uno que está a "cargo de la escuela" pues "se refiere específicamente a las decisiones de gestión sobre los contenidos de ese componente" (SEP, 2016: 61). Por ello este análisis no lo considera y, en cambio, se centra en los dos primeros componentes. El primero de ellos, Aprendizajes clave, se desglosa en Campos formativos y asignaturas, dependiendo de que se trate del nivel preescolar, primaria o secundaria. El inicial de estos Campos formativos se llama Lenguaje y comunicación, incluyendo desde luego al lenguaje, que encabeza la malla curricular. Los dos restantes Campos formativos de este Componente son Pensamiento matemático y Exploración del mundo natural y social.

Iraguirre Ruiz, doi: https://doi.org/10.36799/el.v2i1.55, Volumen 2, número 1, Año 2017, ISSN: 2448-5942 
A su vez, el segundo gran Componente, Desarrollo personal y social, se desglosa en tres Áreas, que serán las mismas para el nivel de preescolar, primaria y secundaria: Desarrollo corporal, Desarrollo artístico y creatividad, Desarrollo emocional. Es evidente que el arte está contenido en este segundo Componente, entre lo corporal y entre lo emocional. Puede constatarse hasta aquí cómo lenguaje y arte aparecen curricularmente distantes uno de otro, a pesar de su conocida naturaleza cerebral común, si bien diferenciados por algunos de sus rasgos singulares. O, quizás, tal organización en el currículo es un reflejo icónico de la conocida diferenciación en cuanto a las funciones controladas por cada uno de los hemisferios.

Sobre este diseño curricular surgen algunos interrogantes. Uno de ellos debe indagar sobre el criterio que coloca a lenguaje y arte como dos cosas separadas, a pesar de la relación evolutiva, biológica y social entre ambos. Otro de los interrogantes es más específico. Y se refiere también al criterio curricular por el cual lo "corporal" y lo "emocional" se presentan como áreas desvinculadas de lo "creativo". ¿Cuáles son las implicaciones pedagógicas, didácticas y sociales de separar en la educación dos elementos cuyo origen y desarrollo evolutivo tienen una naturaleza común basada en la comunicación, la expresión y la cognición?

Esta primera imagen que en la educación se presenta del lenguaje y arte, como objetos separados, llama la atención puesto que la realidad histórica y social muestra siempre imágenes diferentes. Una de éstas es lo que podríamos llamar relación inherente entre lenguaje y arte. La enorme tradición y herencia literaria en castellano es valorada al mismo tiempo como expresión de la razón y la cultura, sintetizada en la sensibilidad de los pueblos correspondientes. En el corpus histórico-literario del México hispanohablante, por ejemplo, desde la Colonia hasta el siglo XX, de Sor Juana a Octavio Paz o Carlos Fuentes, se concentran una buen aparte del desarrollo civilizatorio de una nación y la expresión de su sensibilidad, a pesar de que hoy puedan ser objetadas como formas de racionalidad y sensibilidad elitistas. Más aún, sectores llamados marginales han buscado también sus propias formas de expresión. Géneros literario-musicales como el corrido revolucionario y la época dorada del cine nacional son dos áreas en las que la expresión de la sensibilidad popular halló cauce favorable. En cuanto al mundo indígena, escindido y al margen en múltiples formas de la cultura mestiza, autores como Miguel León Portilla han mostrado una parte significativa, particularmente de la cultura náhuatl, apoyándose justamente en la poesía. Si en todos estos casos hay un continuum entre Razón y Sensibilidad, que 
ilustra el vínculo histórico y social entre lenguaje y arte, característico en el desarrollo civilizatorio tanto como de los individuos, ¿por qué el sistema educativo contemporáneo en México quiere enseñar dichos objetos como entidades desvinculadas?

Más aún. El Campo formativo Lenguaje y comunicación para el nivel de primaria se desglosa en tres asignaturas: Lengua materna y literatura; Español como segunda lengua; Lengua extranjera (inglés). Se infiere que "Español como segunda lengua" es un gesto de atención por parte de la política educativa dirigido a los niños indígenas de México, pero el mismo desglose no aparece para el nivel de preescolar ni se mantiene para el de secundaria. ¿Acaso la población meta es capaz de aprender "Español como lengua materna" solo durante la educación primaria? O bien, ¿siendo hablante nativo de una lengua indígena, se aprende lo suficiente del castellano para la vida durante los seis años de educación primaria? Hay una tercera posibilidad: ¿los niños indígenas no requieren desarrollar habilidades lingüísticas en español como medio de socialización durante su educación preescolar ni secundaria? Esta inconsistencia del currículo se corresponde fielmente con el vacío que representa el nivel de secundaria en el sistema educativo para la población de estudiantes indígenas en el país (INEE, 2016).

Respecto al Componente curricular en que se ubica el arte, el panorama es todavía más delicado. Hemos visto que el Desarrollo personal y social se desglosa en tres Áreas: Desarrollo corporal (que debemos entender como Educación Física); Desarrollo artístico y creatividad (Educación Artística) y Desarrollo emocional (que debemos ver como innovación curricular en la Educación Básica). Si se piensa en tareas o situaciones cotidianas de un niño mexicano típico, como realizar un encargo doméstico, escolar o social, observaremos sin problema que en la ejecución de la actividad se involucra invariablemente lo corporal, la creatividad y lo emocional. Pero cuando se trata de eventos esencialmente recreativos, como la realización de juegos colectivos, la participación en alguna celebración festiva escolar o familiar, la organización sistémica de los niños involucrando cuerpo, creatividad y emoción resulta siempre una experiencia digna de registro. La espontaneidad, autenticidad y eficacia vivencial con que los pequeños ejecutan dichas tareas es de tal fuerza integradora que casi siempre las prefieren por sobre otras responsabilidades, incluidos desde luego los Aprendizaje clave de la propia escuela, que aparecen como prioritarios en el currículo. Una vez más: ¿por qué la escuela querrá enseñar 
de manera fragmentaria la experiencia que cotidianamente se presenta como vivencia integrada? O ¿ंserá otro el propósito de la escuela?

\section{ARTE, LENGUAJE Y SOCIEDAD: LA CULTURA DOCENTE}

¿Cómo se forman profesionalmente los docentes que deben enseñar lenguaje y arte, y cuál es su concepción sobre ambos objetos de enseñanza? Para responder esta pregunta hemos de asomarnos a otro plano del currículo: el de la formación docente en los niveles de preescolar, primaria y secundaria. También serán útiles algunos testimonios de una pequeña muestra de docentes desempeñándose en secundaria, en Hermosillo, Sonora.

El modelo curricular de formación docente para Educación Básica vigente en México (2016) integra el nivel de preescolar y primaria en un mismo plan de estudios, a nivel nacional. En el nivel de secundaria hay un plan de estudios diferente cuyo diseño e implementación queda a cargo de la autoridad educativa de cada entidad federativa. Para este análisis vamos a centrarnos en el peso y valoración que se da a los componentes curriculares relacionados con lenguaje y arte en ambos planes de estudios.

\subsection{PERFIL PROFESIONAL DOCENTE EN PREESCOLAR Y PRIMARIA}

En el plan de estudios de preescolar y primaria, de un total de 55, seis cursos corresponden a Lenguaje (cuatro para Español y dos para Inglés); dos a Tecnologías de la Información y la Comunicación (TIC) y dos a Educación Artística. Se contabilizan en este análisis los cursos de TIC debido a que el currículo los coloca junto a Lengua adicional y debido también a que el propio currículo lo incluye en el Camp formativo Lenguaje y comunicación. Nótese en la diferencia de la cantidad de cursos la importancia que se otorga a cada uno de los campos de análisis, cuyas razones podrían ser claras en el plan de estudios.

\subsection{PERFIL PROFESIONAL DOCENTE EN SECUNDARIA}

Considerando que en el nivel de secundaria el modelo didáctico opera con base en el criterio de la especialidad disciplinar, el perfil docente incluye 22 cursos de Formación específica

Iraguirre Ruir, doi: https://doi.org/10.36799/el.v2i1.55, Volumen 2, número 1, Año 2017, ISSN: 2448-5942 
por especialidad, de un total de 45. Adicionalmente a los 22 cursos de especialidad en Español, debe añadirse un curso de Expresión Oral y Escrita (EOE), común a todas las especialidades, por considerar que en dicho curso se desarrollan habilidades necesarias para el resto de las áreas de formación.

Pero en el estado de Sonora, para el nivel de secundaria, la Escuela Normal Superior del Estado, en su programa de Licenciatura en Educación Secundaria, no incluye entre su oferta la Especialidad en Arte o Educación Artística como sí lo hace para las restantes especialidades del currículo, incluido Español. ¿Cómo se forman en el estado de Sonora los docentes de secundaria en la Especialidad de Artes? Como respuesta se localizan dos ofertas. Una en el Instituto de Formación Docente del estado de Sonora (IFODES); la segunda en el Centro Regional de Formación Docente e Investigación Educativa (CRFDIE). La primera oferta se titula "Especialidad en Competencias para la Enseñanza de las Artes en la Educación Básica". Su objetivo establece

Conocer los enfoques, principios pedagógicos, metodología y las orientaciones didácticas de la Reforma Integral de la Educación Básica en la enseñanza de las artes en los tres niveles de educación básica, con la finalidad de desarrollar competencias de intervención docente que a su vez promuevan las competencias para la vida y el logro del perfil de egreso de sus alumnos (IFODES, 2017:

http://www.ifodes.edu.mx/oferta_detalle.php?op=ECEAEB).

La estructura curricular de esta Especialidad contiene 200 horas distribuidas en cinco módulos: La enseñanza de las artes en el marco de la Reforma Integral de Educación Básica (40 horas); Competencias y estrategias para enseñanza de las Artes Visuales (40 horas); Competencias y estrategias para enseñanza de la Danza (40 horas); Competencias y estrategias para enseñanza del Teatro (40 horas); Competencias y estrategias para enseñanza de la Música (40 horas).

La segunda opción es parte de la oferta del CRFDIE. Se trata de un Diplomado cuyo título es "Educación a través de las Artes".

Iqaguirre Ruir, doi: https://doi.org/10.36799/el.v2i1.55, Volumen 2, número 1, Año 2017, ISSN: 2448-5942 
Tiene como finalidad ofrecer a los participantes una experiencia de desarrollo que permita reconocer la importancia de una intervención educativa articulando la enseñanza de las artes. Este diplomado se fundamenta en el arte, entendido como una actividad culturalmente condicionada mediante la cual los sujetos materializan de forma creativa sus valores, ideologías y visiones del mundo con el fin de transmitirlos a otras personas. No se limita a establecer un contacto efímero ni fragmentario entre las expresiones artísticas y los participantes. Se parte de un análisis inter y trans-disciplinario, que recupera la potencialidad del arte en el desarrollo cultural y de modo particular, en la educación. Se valora la experiencia comunitaria dada por el arte y se coloca como propulsor del desarrollo individual, favoreciendo la interacción entre los sujetos (CRFDIE, 2017; http://www.crfdies.edu.mx/sitiov2/index.php?r=sitio/arte)

La estructura curricular está integrada de una Inducción y cuatro módulos. La Inducción consiste en Introducción a la perspectiva pedagógica y estética del programa; el módulo 1 se titula Acercamiento a las artes; el módulo 2 es el Eje básico de las disciplinas artísticas; el módulo 3 se llama Criterios y técnicas de producción y análisis; por último, el módulo 4 es una Intervención educativa con un carácter Transversal.

En cuanto a los destinatarios, la Especialidad del IFODES está dirigida a docentes en servicio de preescolar, primaria o secundaria; mientras que el Diplomado del CRFDIE está dirigida a "Docentes de todos los niveles educativos y a estudiantes de licenciatura en educación o áreas afines".

Independientemente del valor pedagógico y disciplinar que puedan tener tanto la Especialidad del IFODES como el Diplomado del CRFDIE, llama la atención que ambas ofertas no sean parte de la formación docente en secundaria, junto al resto de Especialidades de dicho nivel educativo. ¿Cuáles son las razones por las que el modelo de formación docente en secundaria no incluye al arte como una de sus Especialidades? ¿Qué implicaciones tiene que la formación de docentes en este campo ocurra como estrategia de capacitación separada del plan de estudios estandarizado?

Iraguirre Ruir, doi: https://doi.org/10.36799/el.v2i1.55, Volumen 2, número 1, Año 2017, ISSN: 2448-5942 


\subsection{ENFOQUE EPISTEMOLÓgICO SOBRE ARTE-LENGUAJE Y PERFIL DOCENTE}

Acerca de la concepción mutuamente excluyente que el currículo asume entre lenguaje y arte, a pesar del origen humano de ambos y a pesar también de su consistente integración vivencial en la vida cotidiana, ya hemos discutido brevemente al principio de este texto. Pero la separación artificial no se detiene en la visión que se tiene de ellos como objetos de aprendizaje. En los diferentes diseños curriculares se observa que no solo existe una escisión entre arte y lenguaje como asignaturas, sino que además cada uno de dichos objetos es fragmentado internamente como resultado de las exigencias curriculares, las cuales siguen respondiendo a una lógica disciplinar especializante.

Respecto al lenguaje no es el caso que la enseñanza del español como lengua materna, o el inglés como lengua extranjera, deban ser enseñadas haciendo a un lado la complejidad inherente a los procesos propios de cada lengua, acentuada gracias a la diversidad socio-cultural en el país. Se trata de provocar la siguiente reflexión. En tanto que ambas son objetos lingüísticos, español como lengua materna e inglés como lengua extranjera, su naturaleza tiene un fundamento común sobre el que los docentes de cada asignatura deberían tener una profunda conciencia y, en conjunto, convertirla en materia común de trabajo pedagógico. Una acción como ésta tendría que incluir, desde luego, el fenómeno de las lenguas indígenas, cuya problemática particular complejiza de modo extraordinario el panorama de la enseñanza y aprendizaje del lenguaje. Al hacerlo, habría necesidad de dimensionar que la enseñanza de lengua indígena en México (como en grandes áreas del mundo) es un asunto no sólo lingüístico ni tampoco ubicado exclusivamente en lo pedagógico. Fuertes razones de orden ideológico, histórico y político, enmarañan la reflexión sobre la coexistencia del mundo mestizo y los mundos indígenas en un mismo territorio. Así que enseñar lengua entre las comunidades escolares, tanto mestizas como indígenas, tendría que pasar por enfrentar algunos de los significados de tal circunstancia, al menos como una forma de avistar la penosa situación reportada en el Panorama Educativo de la Población Indígena (INEE; 2016).

En general

Iraguirre Ruir, doi: https://doi.org/10.36799/el.v2i1.55, Volumen 2, número 1, Año 2017, ISSN: 2448-5942 
Es interesante observar cómo la escuela oficial latinoamericana, pese a ese afán histórico castellanizador y homogeneizador que la ha caracterizado, $[. .$.$] no ha logrado$ desarrollar programas de estudio, metodologías y técnicas adecuadas para apoyar el aprendizaje del castellano por parte de los educandos vernáculohablantes. Pese al tiempo transcurrido desde la llegada del castellano a estas tierras, su enseñanza sigue siendo una de las áreas más desatendidas.

Ello ocurre aun cuando se trata de la lengua común de la región y del único idioma capaz de constituirse en lengua de relación intercultural no sólo entre países sino, y sobre todo, entre los distintos pueblos indígenas de la región. Lo cierto es que ese afán igualador y negador de las diferencias no nos ha permitido caer en cuenta de que estamos frente a poblaciones con necesidades de aprendizaje particulares a las cuales es menester responder de forma diferenciada (López, 2003: 39-40).

Reconocer la complejidad que involucra la enseñanza de lengua en un escenario sociocultural como México, debería ser un reflejo de la voluntad política tendiente a atender las necesidades comunicativas, expresivas y cognitivas basadas en el componente lingǘstico del desarrollo humano. También, de modo concreto, debería traducirse en la formación de docentes cuyo perfil incluya esta complejidad lingüística y cultural.

Por lo que toca al arte el panorama no es menos complicado. ¿Cuál es la definición de arte en el currículo de educación básica, y en qué medida esta definición recupera la diversidad cultural del país, si eso fuera parte del propósito? ¿De qué forma se integran en el currículo expresiones como bellas artes, arte popular, artesanías? ¿Cuáles son las expresiones artísticas a las que los niños mexicanos - mestizos e indígenas- tienen acceso de manera que dichas expresiones puedan ser materia de aprendizaje en la escuela? ¿Cuál debe ser el perfil docente más indicado para enseñar un conjunto de prácticas cuya naturaleza está diferenciada entre lo kinésico-corporal, lo perceptual, lo expresivo acústico-visual, lo vocal, y que influyen de manera directa en el desarrollo de la subjetividad?

Cuando en la oferta de capacitación de docentes de arte, como la del IFODES y la del CRFDIE, mostradas arriba, observamos la presencia de campos artísticos como artes visuales, danza, música y teatro en una relación adyacente, es inevitable preguntarse con qué frecuencia

Izaguirre Ruiz, doi: https://doi.org/10.36799/el.v2i1.55, Volumen 2, número 1, Año 2017, ISSN: 2448-5942 
los niños socialmente medianos de Sonora tienen la oportunidad de presenciar expresiones profesionales como las señaladas. Pero sobre todo, es inevitable preguntarse por la estrategia didáctica a través de la cual dichas expresiones encuentran integración en la experiencia vivencial cotidiana de los destinatarios, sean éstos los estudiantes o bien los mismos docentes en formación.

\section{ARTE Y LENGUAJE EN EL CURRÍCULO}

Como resultado de las concepciones de arte y lenguaje en el currículo y entre los docentes, tenemos hacia el final de esta reflexión dos objetos que comparten dos características visibles: desnaturalización y fragmentación. En efecto, arte y lenguaje muestran estos rasgos como consecuencia de dos procesos comunes. Son objetos desnaturalizados, primero porque aparecen como cosas artificiales en tanto que la imagen que se registra de ellos en el currículo es una construcción producto de las exigencias administrativas en la educación; como consecuencia, la imagen curricular de ellos difícilmente corresponde con la realidad. Este carácter artificial provoca que arte y lenguaje sean enseguida culturalmente ajenos en la escuela para amplios sectores de estudiantes, por ejemplo, cuando se enseñan reglas gramaticales o bien aspectos técnicos sobre la composición gráfica, sin mostrar significados específicos de estos objetos para los estudiantes.

Además, arte y lenguaje son objetos fragmentarios. Primero en cuanto a su propia naturaleza, que ha sido descompuesta en Campos formativos y éstos a su vez en Asignaturas o Áreas, según su posición en el currículo. Lo anterior da como resultado un aprendizaje en el que el sujeto registra, en el mejor de los casos, pedazos de objetos cuya integración entre ellos prácticamente no ocurre, mientras que la relación de estos fragmentos con la realidad resulta falsa o débil.

Desnaturalización y fragmentación de arte y lenguaje en el currículo son, por otra parte, dos rasgos que se retroalimentan de modo permanente gracias a diversos mecanismos. Dos de ellos son el propio currículo como fuente de conceptualización y los docentes como reproductores de dicha conceptualización. En la práctica escolar hay un extenso repertorio de actividades que dan cuenta de la relación que dialécticamente desnaturaliza y fragmenta a arte y

Iraguirre Ruir, doi: https://doi.org/10.36799/el.v2i1.55, Volumen 2, número 1, Año 2017, ISSN: 2448-5942 
lenguaje. Escribir listas de palabras aisladas entre sí; declamar durante los lunes cívicos; enseñanza reiterativa del verbo To Be; resolver ejercicios de gramática; practicar en Sonora un baile de Chiapas, sin dar contexto al acto de bailar; hacer un dibujo "libre", son algunas tareas que muchos docentes, en su afán por enseñar arte o lenguaje, pero sin un propósito específico claro (Izaguirre, Ruíz, 2016), siguen implementando para responder a una exigencia curricular en la escuela. Puesto que estos docentes han sido profesionalmente formados dentro de su propio currículo, no hay forma de deshacer el círculo.

\section{HACIA LA RECUPERACIÓN DEL SUJETO}

Hemos visto que arte y lenguaje han surgido y coexisten en las coordenadas de la evolución y del desarrollo. Decir que cada uno de ellos se genera en uno de dos hemisferios cerebrales y que éstos por su parte controlan ciertas funciones del comportamiento, es decir una verdad científica cuyas interpretaciones con frecuencia da lugar a equívocos o verdades parciales. Una de dichas verdades parciales es el establecimiento de relaciones unívocas entre LenguajeRazón, por un lado, y entre Arte-Sensibilidad, por otro. Esta definición aislacionista quiere dar la idea de que el sistema neuronal opera como un programa especializado, pero en ella se desconocen las complejas relaciones de interconectividad; luego esta definición se traduce en una visión fragmentaria del sujeto. Por más que la especialización hemisférica en los seres humanos sea una de las conquistas evolutivas más preciadas, vale la pena dimensionar sus interpretaciones cuando se trata de educación.

En los procesos de aprendizaje, incluidos desde luego aquellos de tipo escolar, el sujeto experimenta siempre la realidad de un modo integrado, lo que no evita que eventualmente operen las funciones especializantes del cerebro por ejemplo para discriminar el matiz de un color o el timbre de un sonido, pero sólo bajo condiciones de experiencia específicas. Por lo tanto, insistir en un currículo especializado y especializante es por un lado mantener la imagen de un sujeto fragmentado y por otro restringir la experiencia como fuente de aprendizaje. Aquí se propone, aunque hay que decir que no con originalidad, que la experiencia integrada de la realidad se corresponde con una forma distinta de aprendizaje, en la cual racionalidad y afectividad ya no aparecen sólo como funciones cerebrales especializadas, sino involucradas en un sistema complejo de relaciones subjetivas e intersubjetivas. Algunos de los procesos psico-

Iraguirre Ruir, doi: https://doi.org/10.36799/el.v2i1.55, Volumen 2, número 1, Año 2017, ISSN: 2448-5942 
emocionales más reconocidos en esta concepción del aprendizaje son percepción, apreciación, expresión, cognición. Entre estos procesos, yendo progresivamente de la percepción a la cognición, hay un ordenamiento progresivo que va de lo simple a lo elaborado, para luego volver a empezar en lo simple, pero en un nivel reorganizado que obliga a reestructurar el nivel previo. Así de modo recurrente. A través de ellos el sujeto experimenta la realidad y la organiza de acuerdo con necesidades de diversa naturaleza, que obedecen a sus diferentes ámbitos de desempeño.

La experiencia integrada como premisa educativa - más concretamente como argumento de un modelo curricular- la hemos planteado en otras oportunidades tomando como pretexto precisamente al arte y al lenguaje (Carrillo Vázquez et al. 2012), no en su concepción de asignaturas mutuamente aisladas, sino como fuentes vivenciales en la cotidianidad. Aquella donde arte, lenguaje, con toda su complejidad convergen azuzando a los individuos en su necesidad de percepción, expresión y cognición, sobre el mundo y sobre sí mismos.

\section{REFERENCIAS BIBLIOGRÁFICAS}

Carrillo Vázquez, Yolanda, Hogla Febe Corrales Alday, Juan Crisóstomo Izaguirre, Esteban Moreno Soto, Mario Moreno Zazueta, María del Carmen Valdez Almada, Leticia Teresita Varela Ruíz. «Diplomado: Educación a través del arte.» Diciembre de 2012.

Collingwood, R. G. Los principios del arte. México: Fondo de Cultura Económica, 1985.

INEE. Panorama educativo de la población indígena 2015. México: Instituto Nacional para la Evaluación de la Educación/Fondo de las Naciones Unidas para la Infancia, 2016.

Izaguirre, Ruíz Juan Crisóstomo. Arte y escuelas secundarias en Hermosillo. Relaciones entre curriculo, cultura docente y aprendizaje. Tesis de doctorado sin publicar. Culiacán: Universidad Autónoma de Sinaloa, 2016.

López, Luis Enrique. «¿Dónde estamos con la enseñanza del castellano como segunda lengua en América Latina?» en Jung, Ingrid y Luis Enrique (Compiladores) López. Abriendo la escuela. Lingüistica aplicada a la enseñanza de lenguas. Madrid: Morata, 2003. Págs. 39-71.

Iraguirre Ruir, doi: https://doi.org/10.36799/el.v2i1.55, Volumen 2, número 1, Año 2017, ISSN: 2448-5942 
SEP. El modelo educativo 2016. El planteamiento pedagógico de la Reforma Educativa. México: Secretaría de Educación Pública, 2016.

- Programa de estudio 2011. Guía para la educadora. Educación básica preescolar. México: Secretaría de Educación Pública, 2013.

. Propuesta curricular para la educación obligatoria 2016. México: Secretaría de Educación Pública, 2016.

Shiner, Larry. La invención del arte. Una historia cultural. Barcelona: Paidós, 2004.

Izaguirre Ruiz, doi: https://doi.org/10.36799/el.v2i1.55, Volumen 2, número 1, Año 2017, ISSN: 2448-5942 